\title{
The Research of the Detecting System for the Battery Cycle Life of the Train
}

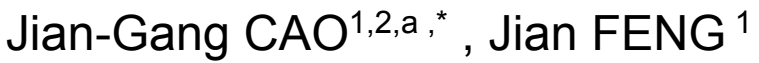 \\ ${ }^{1}$ School of Information Science \& Engineering, Northeastern University, Shenyang Province , \\ China. \\ ${ }^{2}$ CRRC Qingdao SIFANG CO.,LTD. Engineering Laboratory, Qingdao Province, China. \\ acaojiangang10@163.com
}

\begin{abstract}
Keywords: .Battery management system; artificial neural network ; Cycle life; state of charge (SOC) Abstract. The train storage batteries are mainly providing power for train control system and emergency ventilation system. The performance of the vehicle battery status directly affects the safety of the train. Because the cell of batteries is difference, leading to a sharp drop in battery performance and cycle life shortened. Based on the hardware and software platforms of American national instruments(NI), application of artificial neural network algorithm, researching and developing the online detection system of the train battery life. Implementation on the performance of battery state detection and battery life estimates. When using the system on one storage battery, the result shows that the system has some characteristics of simple operation, accurate testing, stability and friendly interface.
\end{abstract}

\section{Introduction}

The Battery is widely used in train auxiliary power system. The Battery is used to supply power for the train lighting system, the emergency ventilation system, the control system and the vehicle signal system. The traditional method of train battery maintenance, including the battery appearance testing, the battery open circuit voltage detection, the battery charge and discharge curve detection, but those methods are unable to predict the performance of the battery and subsequent residual life. At present, the performance of the battery is mainly relying on increasing the number of maintenance or replacing the new batteries.

According to the research on vehicle battery life detection technology, detection system development life battery, realize real-time monitoring and prediction of the performance of the battery life of the battery, and protect the safety of railway operation, optimization of maintenance procedures, reduce the replacement frequency, is very important to strengthen the effective management of the battery, but also has very important social and economic benefit [1-3].

This paper introduces the method of state of charge of the application of BP neural network forecasting battery, and introduces the design process of software and hardware detection system for battery life, the application of the developed testing system to test.

\section{Prediction Model of Battery Charging State}

At present, the common methods of battery charge state detection are [4-6]: density method, open circuit voltage method, timing discharge method, conductance measurement method, and temperature measurement method and so on.

These methods mainly through the detection of battery voltage, current and charge discharge rate and other related parameters to infer, However, the remaining capacity of the battery is affected by many factors, such as temperature, discharge current, battery degradation and so on. it is difficult to establish the mathematical model by using the traditional method, and it is difficult to model accurately. Neural network has a high degree of nonlinearity, but also has the ability to deal with the knowledge of thinking, learning, memory; function approximation performance is strong, more conducive to solve the remaining battery life prediction. 
In this paper, an improved BP neural network ( $\mathrm{LM}$ algorithm) is used to build the SOC prediction model. The following is the process of building model:

Assuming that there are $\mathrm{P}$ training samples, that is $\mathrm{P}$ input and output pairs

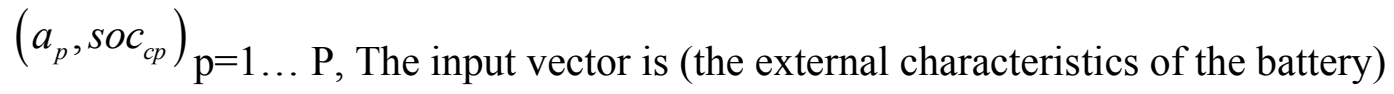

$a_{p}=\left(U_{P}, I_{P}\right)^{T}$

Target output vector (test)

$s o c_{s p}=\left(s o c_{s p 1}, \ldots, s o c_{s p n}\right)^{T}$

Network output vector for (model training):

$\operatorname{soc}_{c p}=\left(\operatorname{soc}_{c p 1}, \ldots, S O c_{c p n}\right)^{T}$

BP network, the network contains 3 layers, with the input layer of the 2 nodes, with the hidden layer of the 12 nodes, with the output layer of the 1 node. As shown in Figure 1, BP neural network based on the neural network prediction model SOC.

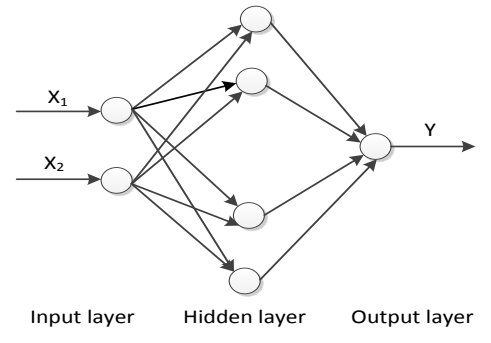

Fig.1 the SOC prediction model based on BP neural network

The input layer selects the discharge current of the battery and the discharge voltage of the battery as the input vector. Through the MATLAB simulation, different training algorithms are used to simulate, and finally, the LM algorithm is chosen to obtain the mean square error of 0.0013.The hidden layer nodes and output nodes use Transing activation function, and the output layer uses Purelin linear activation function.

\section{Application of Artificial Neural Network Algorithm}

And in the node editing artificial neural network algorithm MATLAB program, and run in LABVIEW, Using MATLAB Script nodes to achieve the process of artificial neural network algorithm is as follows:

1)New LABVIEW VI, and add MATLAB Script node in the block diagram program;

2)In the MATLAB Script node to add BP algorithm code, add the corresponding input / output parameters on the node, as shown in figure 2;

3) In LABVIEW VI on the front panel to add the corresponding child controls, and add the relevant input parameters, the output control.

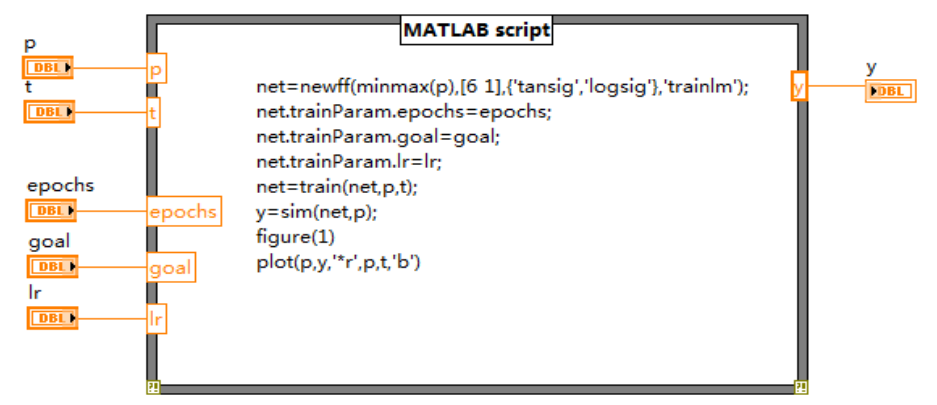

Fig.2 Application of improved BP algorithm 


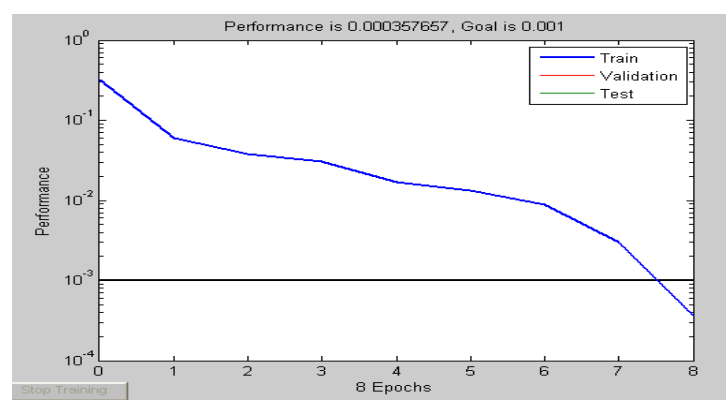

Fig.3 The network training error curve

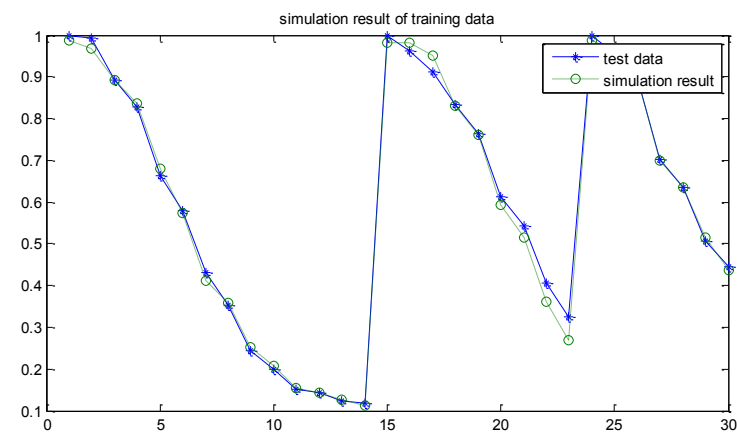

Fig.4 The battery SOC approximation curve

\section{Design of on Line Detection System for Battery Life}

Battery life online testing system structure diagram shown in figure 5, The system is mainly based on the National Instrument Company (NI) hardware and software platform development, Application of LEM sensor to collect battery charging and discharging voltage and current signal, The high precision signal conditioner is used to convert the small current signal output by the sensor into the voltage signal, Then the acquisition module NI6366 is used to collect the acceptable voltage signal. The data are analyzed and processed on the upper computer, and the application of the algorithm is also discussed. Real time detection of battery current, voltage and SOC value is normal. The signal transfer box configuration is shown in table 1 .

Tab.1 The signal switching box configuration tales

\begin{tabular}{l|l|l|l}
\hline $\begin{array}{l}\text { MeasuringSensor } \\
\text { range }\end{array}$ & $\begin{array}{l}\text { Output } \\
\text { type }\end{array}$ & $\begin{array}{l}\text { Sampling } \\
\text { sesistance }\end{array}$ \\
\hline $0-20 \mathrm{~V}$ & LV25-P & $0-20 \mathrm{~mA}$ & $40 \Omega$ \\
\hline $0-150 \mathrm{~V}$ & AV100-150 & $0-50 \mathrm{~mA}$ & $15 \Omega$ \\
\hline $0-150 \mathrm{~A}$ & LT205-T & $0-75 \mathrm{~mA}$ & $10 \Omega$ \\
\hline
\end{tabular}

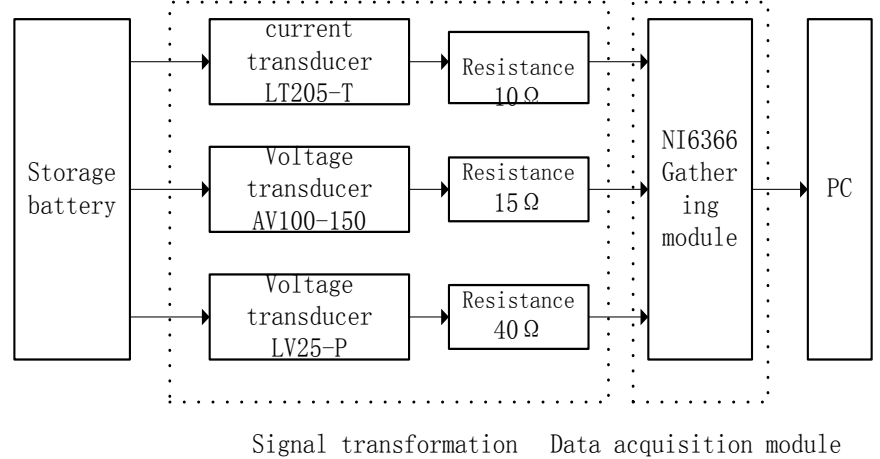

Fig.5 The detection system diagram 
Battery life is terminated according to the following conditions:

1) Battery capacity is less than $80 \%$;

2) Battery discharge time is in line with the requirements;

3) Whether the battery temperature meets the requirements of the battery discharge process;

4) Whether the charging and discharging times are estimated by the remaining capacity of the battery, which meets the requirements of the next running mileage;

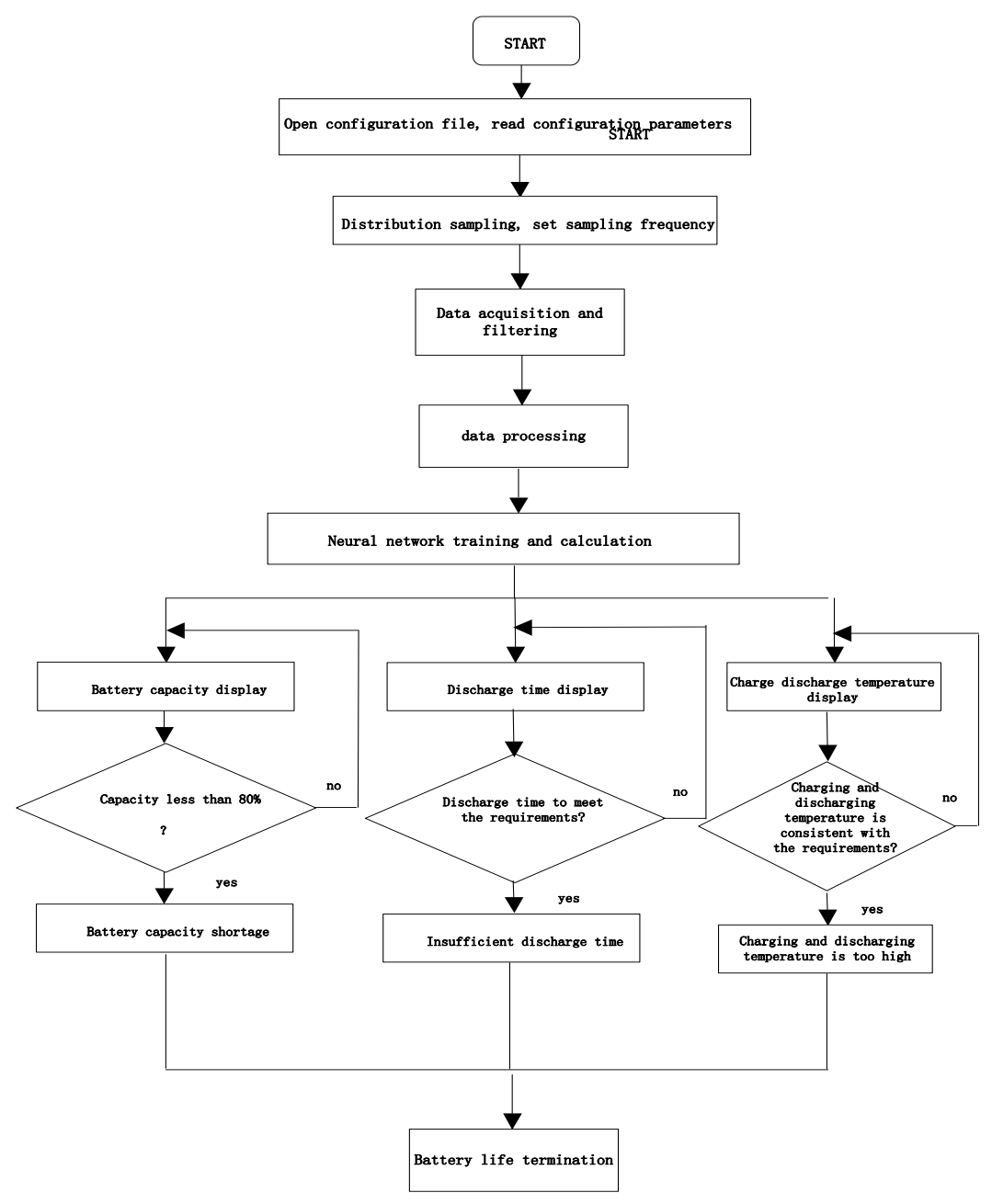

Fig.6 The flow chart of detection system

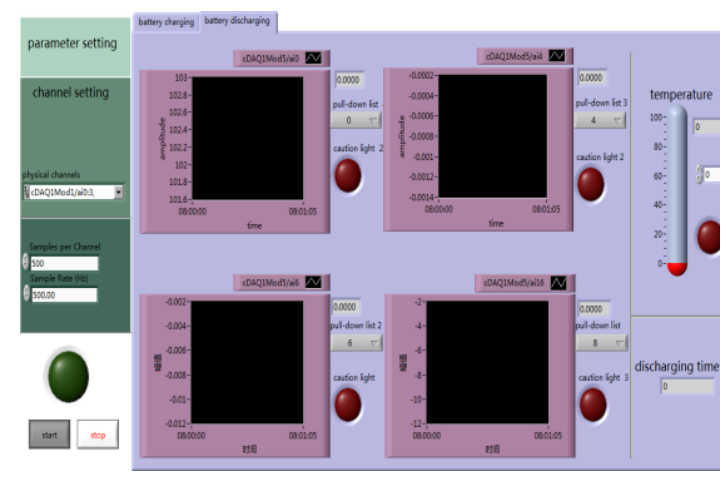

Fig.7 Test system software interface layout 


\section{Experimental Verification}

In this paper, the design of the battery life detection system is applied to the detection of the battery of a certain type of EMU and the long-term tracking test. The obtained characteristic curve of the battery is shown in the following figure, fig8, fig9 and fig10.

The test of alkaline batteries, mainly for train control system, emergency ventilation system to provide power, and has been operating for nearly 2 years. It can be seen from the analysis of the test data that the single voltage of the battery charging process is not higher than $2 \mathrm{~V}$, and the discharge voltage is not less than $1 \mathrm{~V}$, and the discharge time is more than 4.5 hours. Battery charge state is greater than $80 \%$, battery life can also be about 100 cycles. Comprehensive analysis shows that the performance of the battery in good condition, you can continue to use.

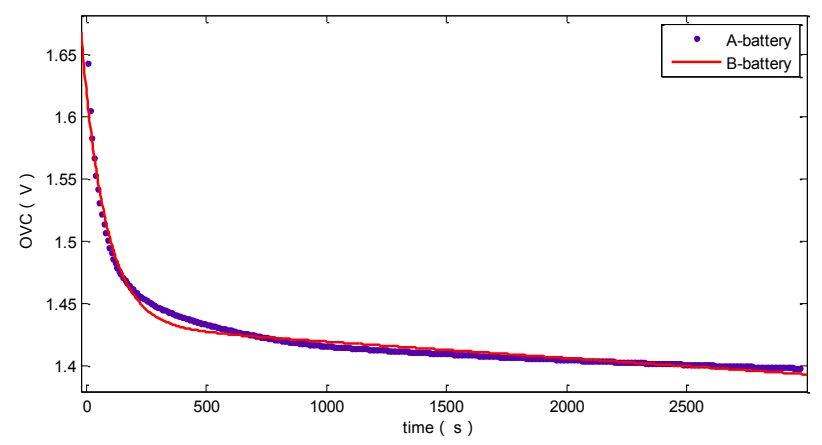

Fig.8 The battery discharge curve

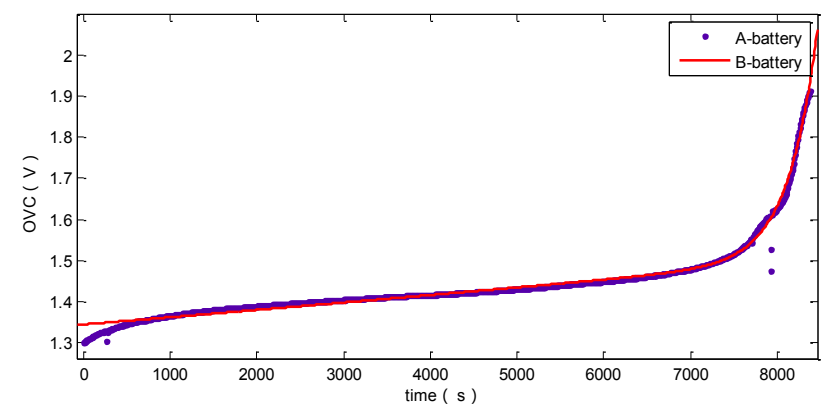

Fig.9 The battery charging curve

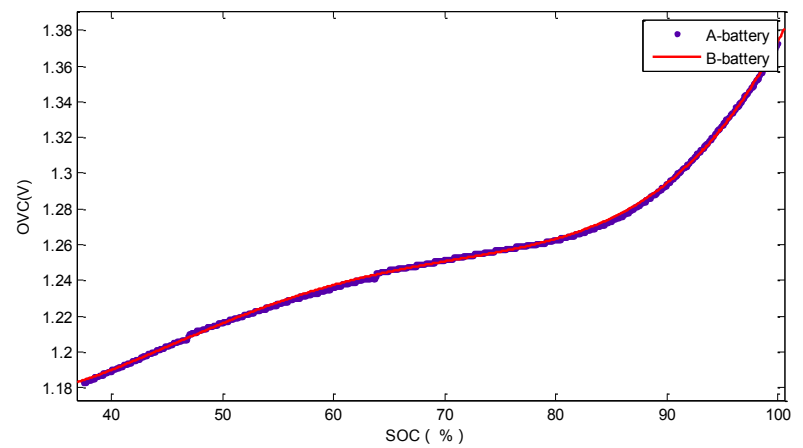

Fig. 10 The battery of SOC curve

\section{Conclusions}

The design and development of the train battery life detection system, remaining battery life algorithm embedded detection device. The battery life decay curve is obtained by estimating the battery charging state, and the remaining life of the battery is estimated. The research and 
development of the detection system, improve the maintenance efficiency, shorten the maintenance cycle, and is of great significance to the safety and economy of the train.

\section{Reference}

[1]Olivares B E, Credo Munoz M A, Orchard M E, et all. Particle-filtering-based prognosis framework for energy storage devices with a statistical characterization of state-of-health regeneration phenomena[J]. IEEE Trans Instrument and Measurement, 2013, 62(2): 364-376.

[2] ALVAREZ ANTON J C, GARCIA NIETO P J, BLANCOVIEJO C, et al. Support vector machines used to estimate the battery state of charge[J]. IEEE Transactions on Power Electronics, 2013, 28(12): 5919-5926.

[3]LUO W L, ZHANG L Q, LV CH, et al. Review on foreign status of life prediction of lithium-ion batteries[J]. Journal of Power Supply, 2013, 45(1):104-144.

[4]WANG D, MIAO Q, PECHT M. Prognostics of lithium-ion batteries based on relevance vectors and a conditional three-parameter capacity degradation mode[J]. Journal of Power Sources, 2013, 239:253-264.

[5]SANCHEZ L, COUSO I, GONZALEZ M. A design methodology for semi-physical fuzzy models applied to the dynamic characterization of LiFePO4 batteries [J]. Applied Soft Computing, 2014, $14: 269-288$.

[6]ANDRE D, APPEL C, SOCZKA-GUTH T, et al. Advanced mathematical methods of SOC and SOH estimation for lithium-ion batteries [J]. Journal of Power Sources, 2013, 224:20-27.

[7]NUHIC A, TERZIMEHIC T, SOCZKA GUTH T, et al. Health diagnosis and remaining useful life prognostics of lithium-ion batteries using data-driven methods[J]. Journal of Power Sources, 2013, 239: 680-688.

[8]ZHOU J B, WANG SH J, MA L P, et al. Study on the reconfigurable remaining useful life estimation system for satellite lithium-ion battery[J]. Chinese Journal of Scientific Instrument, 2013, 34(9): 2034-2044.

[9]Olivares B E, Orchard M E, et all. Particle-filtering-based prognosis framework for energy storage devices with a statistical characterization of state-of-health regeneration phenomena[J]. IEEE Trans Instrument and Measurement, 2013,62(2): 364-376. 